Apidologie, 1974, 5 (1), 21-38.

\title{
FREIE AMINOSÄUREN, QUALITATIV BESTIMMT IN EINIGEN BLÜTEN-WALD-UND MISCHHONIGEN
}

\section{Dêtermination qualitative des acides aminés libres dans quelques miels de fleurs, miels de miellat et miels mixtes}

\author{
Edith KULLMANN
}

Forstzoologisches Institut, Freiburg *

\section{SUMMARY}

\author{
QUALITATIVE DÉTERMINATION OF FREE AMINO ACIDS IN SOME \\ HONEYDEW HONEYS, MIXED HONEYS AND HONEYS FROM FLOWERS
}

The purpose of the analysis was to find out the variety of amino acids in blossom-, forestand mixed-honeys. The qualitative analyses of the honeys for free amino acids were made by paperchromatography. This method enabled to prove 18 amino acids; in the honeys tested, however, a maximum of 16 amino acids was found. Methionine and tryptophan were lacking.

A difference between blossom- and forest-honey came out by the lack of cystine, glycine and histidine in blossom-honey whereat, however, rarely al 3 amino acids mentioned above were lacking in one sample.

Only in Trifolium pratense-honey asparagin could be found. The highest number of amino acids identified in one fixed honey was 15 , this was relevent only in 1 sample of Castanea sativa-honey and in 3 samples of Pinus sylvestris-honey and all mixed-honeys. For the first time the 2-methyl-alanine ( $\alpha$-amino-iso-butyric acid) could be proved in several honeys.

\section{ZUSAMMENFASSUNG}

Zweck der Untersuchung war, das Spektrum der Aminosäuren von Blüten-, Wald- und Mischhonigen kennenzulernen. Die Untersuchungen der Honige auf freie Aminosäuren wurde qualitativ mit Hilfe der Papierchromatographie ausgeführt. Diese Methode gestattete den Nachweis von 18 Aminosäuren; in den Honigen konnten maximal 16 analysiert werden; es fehlten Methionin und Tryptophan.

Ein Unterschied zwischen Blüten- und Waldhonig ergab sich durch das Fehlen von Cystin, Glycin und Histidin bei Blütenhonigen, wobei aber selten alle 3 der genannten Aminosäuren gleichzeitig fehlten.

* Forstzoologisches Institut der Universität Freiburg, 78 Freiburg/Br., Bertholdstrasse 17. 
Allein im Trifolium pratense-Honig war Asparagin auffindbar. Es waren höchstens 15 Aminosäuren in einem bestimmten Honig nachweisbar; dies trifft für 3 Proben der Pinus sylvestris-Honige, 1 Probe des Castanea sativa-Honigs und für alle Mischhonige zu.

Erstmals wurde der Nachweis von 2-Methyl-alanin ( $\alpha$-Amino-isobuttersäure) in verschiedenen Honigen erbracht.

\section{I. - EINLEITUNG}

Honige verschiedener Herkunft (von Nektarspendern bzw. Honigtauerzeugern) werden meist nach mikroskopischer Prüfung ihrer Pollen-, Algenund Pilzsporen- Anteile unterschieden. Die elektrische Leit-fähigkeit sowie der bakterizide Hemmtest (BuCHNER 1966, 1967) ermöglichen weitere zuverlässige Trennungen. Die chemischen Bestandteile des Honigs und Honigtaues wurden im Bereich der Kohlenhydrate gut aufgeklärt (MA URIZIO 1959, 1962).

Die qualitative Bestimmung freier Aminosäuren im Honig sollte zunächst als Hinweis auf geplante quantitative Analysen gelten. 1961, zur Zeit dieser Untersuchungen, war mir nur die Arbeit von Baumgarten und Möckesch (1956) zugänglich. Aus einer Aufstellung über den Nachweis einzelner Aminosäuren im Honig bei Bergner und HaHn (1972) geht hervor, daß Komamine (1960) darüber gearbeitet hat und gleich mir (1961) Asparagin im Honig fand. Weitere Asparagin- Nachweise werden aufgeführt bei Curti und Riganti (1966) sowie Bergner und Hahn (1972). Da ich Asparagin nur im Trifolium pratenseHonig nachweisen konnte, ließ der Vergleich mit den genannten Arbeiten das Ergebnis auch 1972 noch erwähnenswert erscheinen.

\section{II. - METHODIK}

\section{1. - Material}

Untersucht wurden

14 Blütenhonige, davon 7 aus Trifolium pratense-Tracht, die bereits pollen- und zuckeranalytisch geprüft waren (MaURizio 1949, 1959);

weitere 7 aus der Sammlung des Tierhygienischen Institutes, Abt. Bienenkunde, Freiburg /i. Br., die innerhalb der Institutsserien pollenanalytisch untersucht waren; diese sind unter Weglassung der Einzel- pollen in Tabelle 1 aufgeführt,

10 Waldhonige, davon 6 aus der vorgenannten Sammlung; diese und die oben erwähnten Proben sind auf ihre bakterizide Wirkung getestet worden (BuCHNER 1966, 1967);

je 2 aus Pinus sylvestris- und Picea abies- Trachtgebieten, jedoch nicht pollenanalytisch kontrolliert,

4 Mischhonige von Imkern; nicht mikroskopisch überprüft,

1 Zuckerfütterungshonig,

2 Honigtaue, die im Labor von der Buchenkrebs-Baumlaus (Lachnus exsiccator Alt. = Schizodryobius pallipes H.tg.) bzw. der Zirbelkiefern-Rindenlaus (Cinaria cembrae Chol.) gewonnen waren. 
TAB. 1. - Pollenanalysen und Werte des Bakterizidtests der Honigproben aus dem Tierhygienischen Institut, Abt. Bienenkunde, Freiburg/Br.

TABL. 1. - Analyse pollinique et valeurs du test d'activité bactéricide des échantillons de miel provenant de l'Institut vétérinaire, section apicole, de Fribourg en Br.

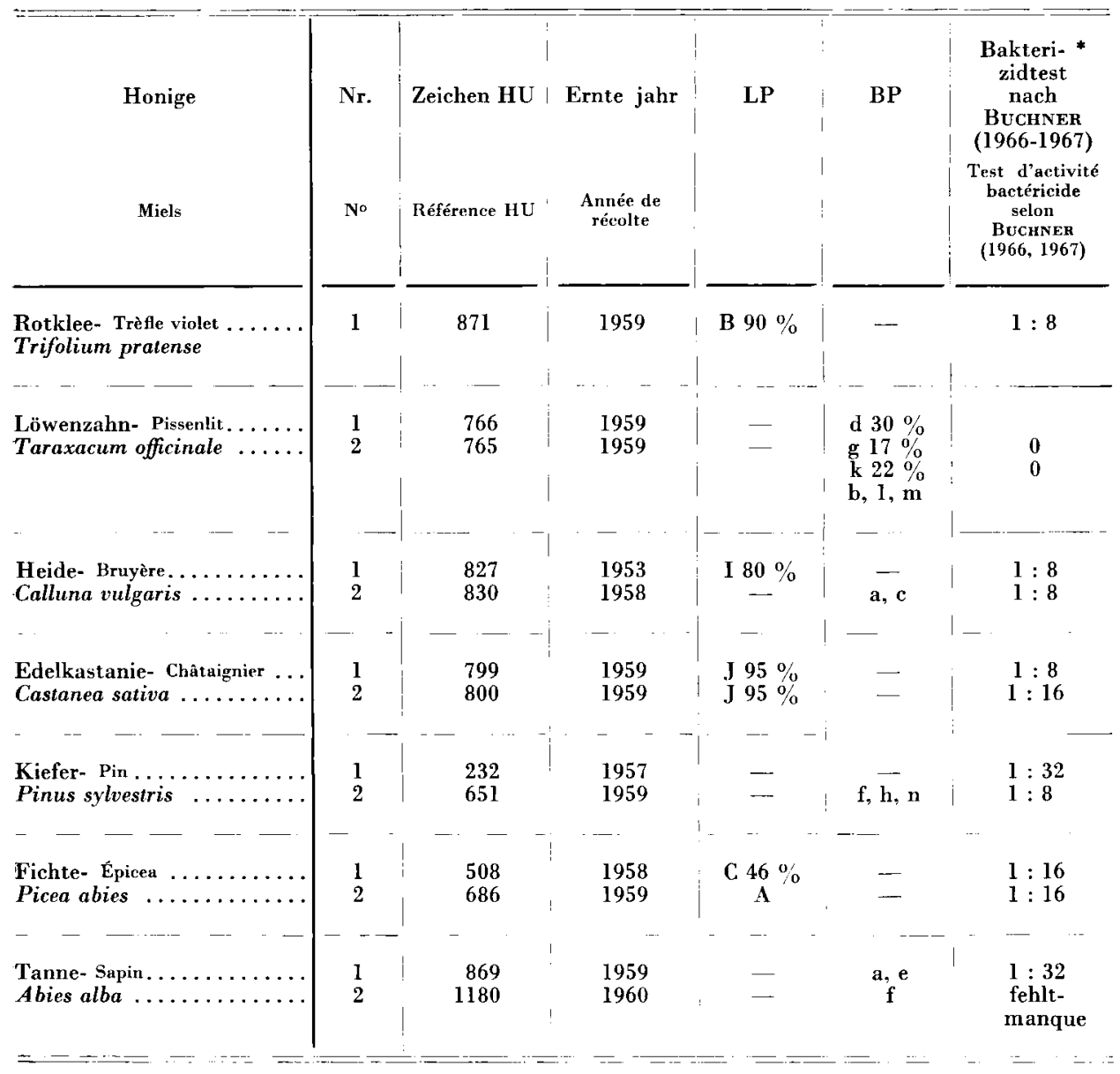


Zu TAB. 1 - TABL. 1 (Explications)

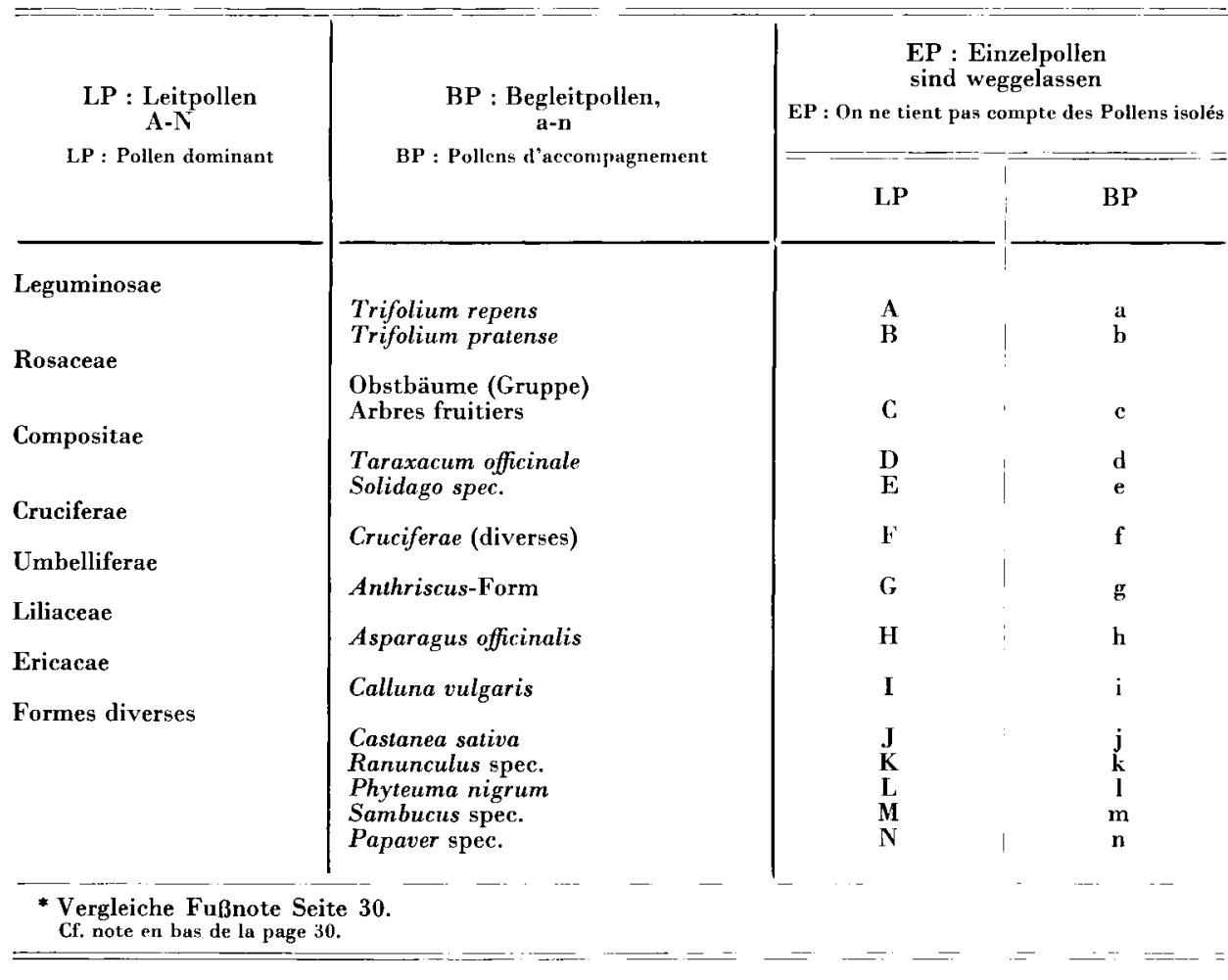

\section{2. - Testsubstanzen}

Die nachstehenden Aminosäuren (Reinsubstanzen von Th. Schuchardt, München und E. Merck, Darmstadt) wurden als 0,5\% \% ige wässrige Lösungen mit $6 \mu l$ fassenden Glaskapillaren als Vergleichssubstanzen aufgetropft.

Die angegebenen Färbungen gelten für die Lösungsmittel Butanol/Eisessig/Wasser, 4/1/1 und 4/1/5 gemischt. Die Färbungen in Phenol sind nicht angegeben.

1) Alanin

2) 2-Methylalanin ( $\alpha$-Amino-iso-buttersäure)

3) Arginin

4) Asparagin

5) Asparaginsäure

6) Cystin

7) Glutaminsäure/ Threonin ungetrennt

8) Glycin (Glykokoll)

9) Histidin

10) Leucin/Isoleucin ungetrennt

11) Lysin

12) Phenylalanin

13) Prolin

14) Serin

15) Tyrosin

16) Valin

17) Methionin

18) Tryptophan

(Ala)
(nicht verze
(Arg)
(Asn)
(Asp)
(Cys-S) 2
(Glu/Thre)
(Gly)
(His)
(Leu/Ileu)
(Lys)
(Phe)
(Pro)
(Ser)
(Tyr)
(Val)
(Met)
(Try)

blau

blau

blau orange grün bis blaugrün rosa bis hellblau blau braun braun blau blau hellblau oliv (grüngelb) blau grau blaubla 8-blau Iila braublau 


\section{3. - Chemikalien und Gerate für}

Säule $=$ Glasrohr m. Hahn (Glaswolle) $30 \mathrm{~cm}$ lang, $0,9 \mathrm{~cm} \varnothing$ Ionenaustauscher I (stark saurer Kationenaustauscher) Austauschkapazität ca 4,5 mval/g, Merck

Äthanol

Salzsäure

Ammoniaklösung

Erlmeyerkolben, Tropftrichter

Destillation $=$ Schliffdest. f. Halbvakuum

Aeromat Standart-Laborkompressor (Saugluft $660 \mathrm{~mm}$ QS)

Durchlaufchromatographie

Butanol-(1) (n-Butylalkohol) f. Chromatographie

Essigsäure min. $96 \%$ ig f. Chromatographie

Sprühreagenz nach Diss. MERTEN.

Ninhydrin, Aceton, 2,4 - Lutidin f. Chromatographie $(0,5 \%$ ige Ninhydrinlsg. in Aceton, je $100 \mathrm{ml} 5 \mathrm{ml}$ Lutidin)

Sprühreagenz nach Cramer (1958).

$(0,2 \%$ ige Ninhydrinlösung in $95 \%$ igem Butanol u. $5 \% 2 \mathrm{~N}$ Essigsäure $)$

Glasgefäße $50 \times 50 \times 20 \mathrm{~cm} \mathrm{mit} \mathrm{Trog} \mathrm{u}$. Einsatz

Aufsteigende Chromatographie, eindimensional

Phenol krist. (org. Phase)

Natriumcyanid gepulvert

f. Chromatographie

Standzylinder ca $40 \mathrm{~cm}$ hoch

Scheidetrichter

Sonstiges : Abzug, Trockenschrank, Eisschrank, Tourenzentrifuge, Handzuckerrefraktometer nach Zeiß, Glaskapillare $6 \mu \mathrm{l}$, Whatman 1 -Papier-Bogen $46 \times 46 \mathrm{~cm}$.

\section{4. - Vorbereitung des Untersuchungsmaterials}

Mittels Kationenaustauscher wurden die Zucker aus der wässrigen Honig-oder Honigtaulösung entfernt. Der Reproduzierbarkeit und der Aufnahmekapazität der Säule wurde durch die Einstellung der Honiglösung auf $20 \%$ Trockensubstanz mit dem Handzuckerrefraktometer nach Zeiß entsprochen.

Die Eiweißfällung wurde durch Zugabe hochprozentigen Äthanols nach DönFeL in LiNSKENS (1959) ausgeführt.

\section{5. - Reinigung und Anreicherung der Aminosäuren am Kationenaustauscher}

In Anlehnung an die Methoden, die G. Zimmermans, Staatsexamenskandidat (1961) im Botanischen Institut der T. H. Darmstadt unter Prof. Ziegler, zur Bestimmung von Aminosäuren in Siebröhrensäften verwendete, verfuhr ich mit allen Honigen und Honigtauen wie folgt :

\section{Zuckereluierung.}

Ionenaustauscher I Merck (stark saurer Kationenaustauscher) mit $300 \mathrm{ml} 10 \%$ iger Salzsäure versetzen, mit $80 \%$ igem Athanol auf pH 6 gewaschen ca 12 Stunden stehen lassen. Die Analysenlösung pH 3 (saurer als Säule) wird innerhalb von I Stunde mit $100 \mathrm{ml}$ Aqua dest. $(0,2 \mathrm{ml} /$ Min.) von den Zuckern befreit und mit Ninhydrin-Reagenz die Aminosäure-freiheit festgestellt.

Aminosäureeluierung.

Aminosäuren mit $300 \mathrm{ml} \mathrm{NH}:$ und $150 \mathrm{ml}$ Aqua dest. in ca 5 Std. eluiert. Bei 600 bis $620 \mathrm{~mm}$ QS Saugluft (Halbvakuum) bis auf $1 \mathrm{ml}$ Volumen eingedampft.

\section{6. - Papierchromatographie}

Ein Eluat liegt vor, enthält alle Aminosäuren.

Durchlaufchromatographie auf $\mathrm{W}_{\mathrm{t}}$-Papier $46 \times 46 \mathrm{~cm}$, Verlust durch Papierpiervorbereitung 10-12 cm. Je Bogen 13 Tropfen, die mit $6 \mu l$ Glaskapillaren aufgebracht wurden. Davon 
sind 2 Tropfen Honiganalysengemisch mit je $30 \mu \mathrm{l}$ pro Tropfen und 11 Tropfen der Testgemische mit $30 \mu \mathrm{g}$ pro Aminosäure. Laufzeit 22 Stunden.

Lösungsmittelgemisch Butanol/Eisessig/Wasser, 4/1/5 trennt besser in Startpunktnähe und $4 / 1 / 1$ in Startpunktferne.

Jede Honigprobe wurde darum mit beiden Lösungsmittelgemischen getestet. Sprühreagenz (Merten 1963) 0,5\% iges Ninhydrin in Aceton mit Lutidinzusatz (je $100 \mathrm{ml} 5 \mathrm{ml}$ Lutidin).

Vor und nach dem Besprühen 30-60 Minuten im Luftstrom aufhängen. Trocknung ca 4 Minuten bei $62^{\circ} \mathrm{C}$.

Als sicher anwesend gilt eine Aminosäure nach 8-17 maliger Wiederkehr. Asparaginsäure, hellgrün gefärbt, verblaßt besonders rasch, dagegen waren Asparagin mit orangefarbenem und Prolin mit olivfarbenem Fleck immer sehr deutlich und anhaltend sichtbar.

Glutaminsäure und Threonin konnten nicht voneinander getrennt bestimmt werden, ebenso war es mit Leucin und Isoleucin.

Die aufsteigende Chromatographie, eindimensional, mit Phenol und Natriumeyanid (Org. Phase) (Cramer 1958) wurde im Standzylinder auf einer Papierrolle (Zone $34 \mathrm{~cm}$ ) ausgeführt. Sie eignete sich nur zur Nachprüfung einzelner Aminosäuren.

\section{III. - ERGEBNISSE}

\section{1. - Aminosäuren im Honig}

In allen 28 untersuchten Honigen aus Blüten-, Wald- und Mischtracht, die als Blüten-, Wald- und Mischhonige aufgeführt sind, konnten qualitativ nachgewiesen werden :

Alanin, Glutaminsäure/Threonin (ungetrennt), Leucin/Isoleucin (ungetrennt), Lysin (mit 2 Ausnahmen), Phenylalanin, Prolin, Serin (mit 3 Ausnahmen), Tyrosin und Valin. Folgende Aminosäuren waren nicht in allen Honigen nachweisbar : 2- Methyl- alanin, Arginin, Asparagin, Asparaginsäure, Cystin, Glycin und Histidin.

Die letztgenannten 3 Aminosäuren eigneten sich durch ihr unterschiedliches Fehlen zur Unterscheidung von Blüten- und Waldhonig.

Asparagin, das Halbamid der Asparaginsäure, wurde zu einem spezifischen Nachweis für Trifolium pratense- Honig.

Von den 18 nachweisbaren Aminosäuren konnten 16 in den Honigen aufgefunden werden. Methionin und Tryptophan bleiben davon ausgenommen.

Die Analyse ergab als Durchschnittszahl aus 14 Proben der Blütenhonige 12 Aminosäuren, aus lo Proben der Waldhonige 14 Aminosäuren und aus 4 Proben der Mischhonige 15 Aminosäuren.

\section{2. - Blütenhonige}

Alle Blütenhonige wiesen im Vergleich zu den Chromatogrammen der Waldhonige eine schwächere Färbung der Aminosäuren auf. Im Blütenhonig 
fehlten Cystin, Glycin, Histidin im Wechsel, jedoch nie alle drei zu gleicher Zeit, außer bei einer Probe von $T$. pratense (Tab. 2, A 534). Von den 14 Blütenhonigen (Tab. 2) fallen die Proben T. pratense A 485 und Castanea sativa HU 800 nicht unter diese Definition; es wurde Cystin, Glycin und Histidin analysiert. Die Aussage gilt für 11 Honige, davon waren 7 mal die Kombination Glycin / Histidin, 2 mal Cystin/Glycin und 2 mal Cystin allein nicht nachweisbar. 2Methyl-alanin ( $\alpha$ - Amino- iso- buttersäure), meines Wissens bisher in Honigen noch nicht festgestellt, ließ sich in 8 von 14 Proben auffinden. Asparagin war nur in $T$. pratense- Honig nachweisbar, und zwar in 6 von 8 Proben.

\section{Honige aus Trifolium pratense-Tracht}

Der erste zur Untersuchung gekommene Honig (Tab. 2, HU 871) aus Boxtal bei Wertheim in Nordbaden (1959) zeigte im Chromatogramm alle Aminosäuren ganz schwach an, deutlich war nur ein orangefarbener Fleck. Zur besseren Identifizierung kam dieser Honig in doppelter Konzentration auf die Säule. Dieser bisher nie erschienene Fleck erwies sich als Asparagin; ihn konnte ich in 6 der $8 T$. pratense- Proben in derselben Deutlichkeit wiederfinden. Um eine Erklärung für die Anwesenheit von Asparagin zu haben, verglich ich Nektare von T. pratense, T. repens und Vicia spec. mit denen einer Senecio spec. und einer Impatiens spec. Da es nur auf diesen einzelnen Nachweis ankam, nahm ich vorbereitetes Chromatographiepapier mit auf die Wiese hinaus. Einmal drückte ich den Nektar der Blüten direkt auf dem Papier aus, zum anderen löste ich mit Aqua dest. den Nektar aus den Blüten heraus und tropfte ihn mit einer Kapillare auf. Die Chromatogramme zeigten bei Nektar von $T$. pratense und $T$. repens sowie von Vicia spec., den Leguminosen, eindeutig Asparagin im Gegensatz zu dem Nektar von Senecio spec. und Impatiens spec., bei denen der Nachweis negativ verlief.

Aus diesen wenigen Untersuchungen an $T$. pratense- Honigen und verschiedenen Nektaren läßt sich nicht sicher schließen, daß bei vermehrtem Stickstoffgehalt, wie er in Leguminosen anzutreffen ist, auch überall Asparagin zu finden sein müßte. Daß aber Asparagin bei einem erhöhtem Stickstoffhaushalt anwesend sein kann, geht aus folgendem Zitat hervor, das ich im Lehrbuch von Strasburger, 1958, S. 238 fand : “ Bei den meisten Pflanzen aber spielt die schon früher genannte Glutaminsäure bzw. Asparaginsäure die Rolle eines Ammoniakentgifters, indem sich die entsprechenden Amide Glutamin und Asparagin bilden, die sich oft in größerer Menge in der Pflanze ansammeln und offenbar auch transportiert werden können (Amidtyp). Auch ihre $\mathrm{NH}_{2}$ Gruppen können leicht zu neuen Synthesen von Aminosäuren wieder abgespalten und übertragen werden - - )

Es lagen 8 T. pratense- Honige zur Untersuchung vor (Tab. 2). Asparagin 


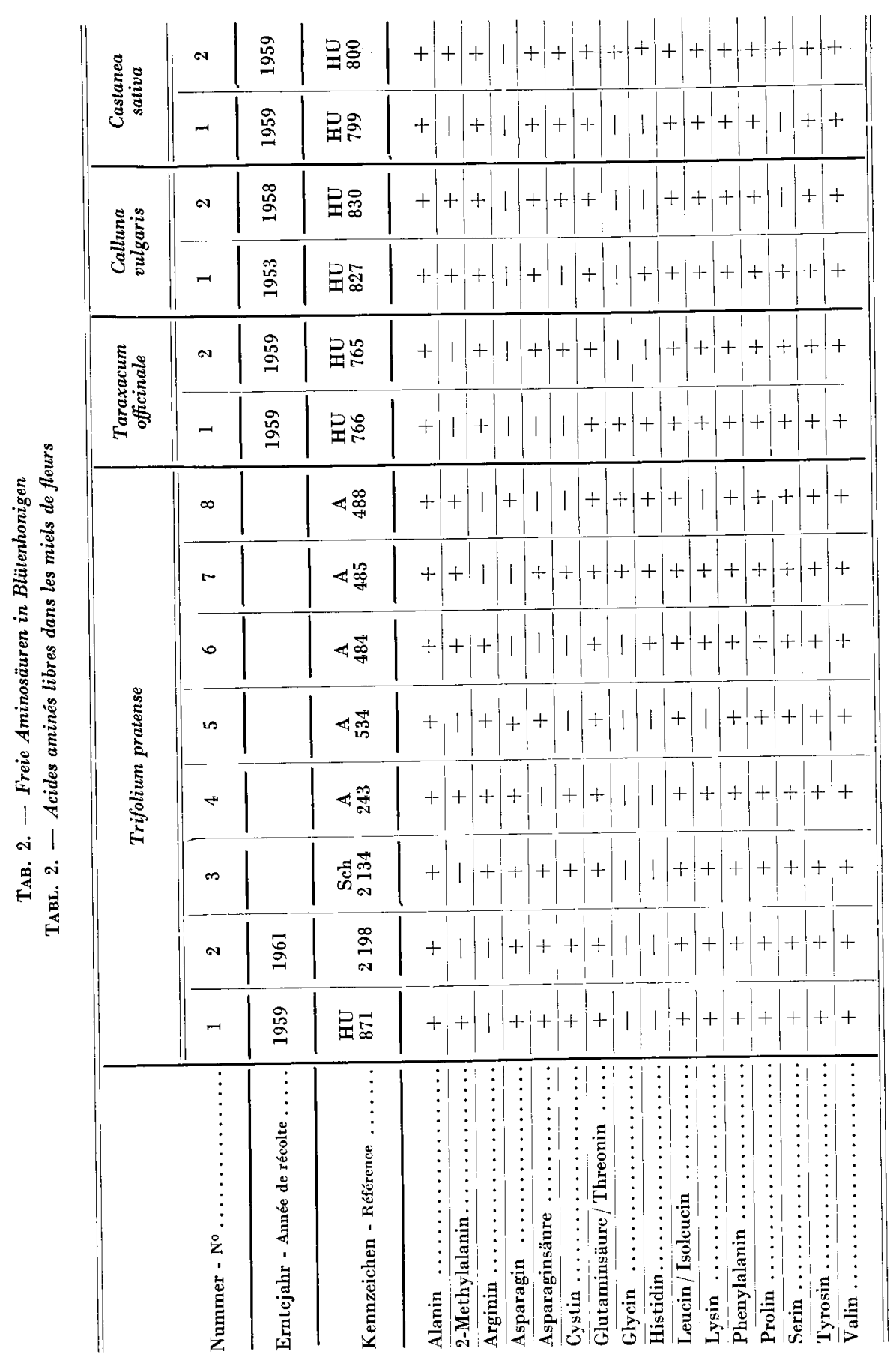


Zu TaB. 2. - TABL. 2. (Explications)

Herkunft der Honige

Origine des miels

\begin{tabular}{|c|c|c|c|}
\hline Trifolium pratense & 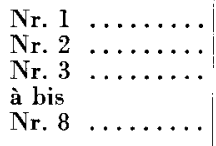 & $\begin{array}{l}\text { Boxtal bei Wertheim, Odenwald } \\
\text { Bern- Liebefeld } \\
\text { siehe } \\
\text { (MAUnizio } \\
1949,1959 \text { ) }\end{array}$ & $\begin{array}{l}\text { Nordbaden Bade } \\
\text { Schweiz Suisse }\end{array}$ \\
\hline Taraxacum officinale & $\begin{array}{l}\text { Nr. } 1 \\
\text { Nr. } 2\end{array} \ldots \ldots \cdots \cdots$ & $\begin{array}{l}\text { Hlertissen } \\
\text { Waldau bei Neustadt }\end{array}$ & $\begin{array}{l}\text { Schwaben Souabe } \\
\text { Südbaden- Bade }\end{array}$ \\
\hline Calluna vulgaris & $\begin{array}{l}\text { Nr. } 1 \\
\text { Nr. } 2\end{array} \ldots \ldots \ldots \ldots$ & $\begin{array}{l}\text { Celle } \\
\text { Celle }\end{array}$ & $\begin{array}{l}\text { Lüneburger } \\
\text { Heide Lande du Lunebourg }\end{array}$ \\
\hline Castanea sativa & $\begin{array}{ll}\text { Nr. } 1 & \ldots \\
\text { Nr. } 2 & \ldots\end{array}$ & $\begin{array}{l}\text { Jockgrimm } \\
\text { Dürkheim }\end{array}$ & $\begin{array}{l}\text { Pfalz- Palatinat } \\
\text { Pfalz- Palatinat }\end{array}$ \\
\hline
\end{tabular}

wurde sowohl in der Probe aus Nordbaden (HU 871) wie in der frisch geernteten Probe (2198) aus Bern- Liebefeld, Schweiz und in den Honigen aus der Sammlung MA Urizio aufgefunden. Aus dieser Sammlung stammen allerdings auch die beiden Proben, denen Asparagin fehlt (A 484 und A 485). Die Probe A 485 wurde schon erwähnt; sie fiel nicht unter die Definition, die sich für die Blütenhonige ergab, sie hebt sich außerdem aus diesem Rahmen noch durch den Gehalt von 14 Aminosäuren heraus. Vergleicht man die T. pratense- Proben mit den übrigen Blütenhonig-Proben, so fällt auf, daß Arginin nur bei $T$. pratenseHonigen fehlt und zwar bei 4 von 8 Proben. 2- Methyl-alanin und Asparaginsäure war jeweils in 3 Fällen nicht nachweisbar; Lysin fehlte 2 mal innerhalb der $T$. pratense-Honige.

Die Durchschnittszahl der gefundenen Aminosäuren beträgt unter Berücksichtigung von Asparagin für T. pratense- Honig allein und für Blütenhonige insgesamt 12,5 .

\section{Honige aus Taraxacum officinale-Tracht}

Die Probe aus Illertissen, Schwaben (Tab. 2, HU 766) wurde ein zweites Mal mit doppelter Ausgangskonzentration über die Säule geschickt. Das Erscheinungsbild zeigte keinen Unterschied, die Nachweise waren intensiver gefärbt. Die Probe HU 765 aus Waldau bei Neustadt im Schwarzwald, Südbaden, hat mit der ersten Probe das Erntejahr (1959), das Fehlen von 2- Methylalanin und den Nachweis von 12 Aminosäuren gemeinsam. Unterschiedlich wiesen sie sich als Blütenhonige aus, indem der ersten Probe Cystin allein und der zweiten Probe die Kombination Glycin/Histidin fehlte. Der T. officinaleProbe HU 766 fehlte außerdem Asparaginsäure, als einzigem der 6 Blütenhonige aus T. officinale-, Calluna vulgaris und Castanea sativa- Tracht. 
Honige aus Calluna vulgaris-Tracht

Die Proben aus der Lüneburger Heide (Tab. 2, HU 827 und HU 830) Ernte 1953 und 1958, unterscheiden sich, indem der ersten Probe die Kombination Cystin/Glycin, der zweiten Probe Glycin/Histidin und außerdem Serin fehlen. So wurden einmal 13, das andere Mal 12 Aminosäuren analysiert.

\section{Honige aus Castanea sativa-Tracht}

Beide Proben sind aus den Edelkastanienbeständen der Pfalz und dem Erntejahr 1959. Die Probe HU 799 weist nur 11 Aminosäuren auf, es fehlen neben der Kombination Glycin/Histidin noch 2- Methyl-alanin und Serin. Im Gegensatz dazu konnten in der Probe HU 800, 15 Aminosäuren, die höchste Zahl der in den Honigen nachgewiesenen Aminosäuren, bestimmt werden.

Dieses Bild ergibt sich sonst nur bei Mischhonigen und in 3 von lo Waldhonigen. Damit fällt diese Probe aus dem Rahmen der Blütenhonige heraus, obwohl die Pollenanalyse $95 \%$ Castanea sativa als Leitpollen aufweist. Es liegt die Vermutung nahe, daß diese Probe Honigtauanteile enthielt. Der angegebene Wert aus dem Bakterizidtest (Tab. 1) beträgt $1: 16$; er unterstützt somit diese Annahme*.

\section{3. - Waldhonige}

Bekanntlich verarbeiten die Bienen den Honigtau, ein Ausscheidungsprodukt von Blatt-Rinden-und Schildläusen, zu Tauhonigen. Die Pflanze ist letztlich immer der Lieferant mit den Inhaltsstoffen der Siebröhrensäfte. Die bienenwirtschaftliche Bedeutung der Honigtauproduktion ist durch zahlreiche Arbeiten, namentlich aus unserem Institut, bekannt (WELLENSTEIN 1958, 1960, 1961, 1967). Letzterer äußerte sich z. B. (1961, S. 199) : « Die Honigtauerzeuger nehmen als Nahrungslieferanten eine Schlüsselstellung in der Waldlebensgemeinschaft ein, deren waldhygienische Bedeutung schwer in Zahlen auzudrücken ist, während sich ihr Gewicht für die Imkerei recht genau in Zahlen angeben läßt. Die mit ihrer Hilfe von den Bienen gesammelten Waldhonige lassen sich heute nach Kiefern-, Fichten-, Tannen-und Lärchenhonigen gliedern und auch medizinisch- therapeutisch von den Blütenhonigen unterscheiden. ” 6 Unterscheidungsmerkmale für Honige aus Blüten-und Honigtautracht führt Buchner (1967) auf; neben der bakteriziden Wirkung ist es die Farbe, das mikroskopische Bild, der Gehalt an Kohlenhydraten und Mineralstoffen s owie die elektrische Leitfähigkeit.

\footnotetext{
* Die Werte aus dem Bakterizidtest geben an, in welcher Verdünnung die Honiglösung den Testkeim Staphylokokkus aureus abtötet. Nach BuchNer (1966) entwickeln Honigtauhonige im Durchschniti eine bedeutend höhere antibakterielle Wirkung als Bliitenhonige.
} 


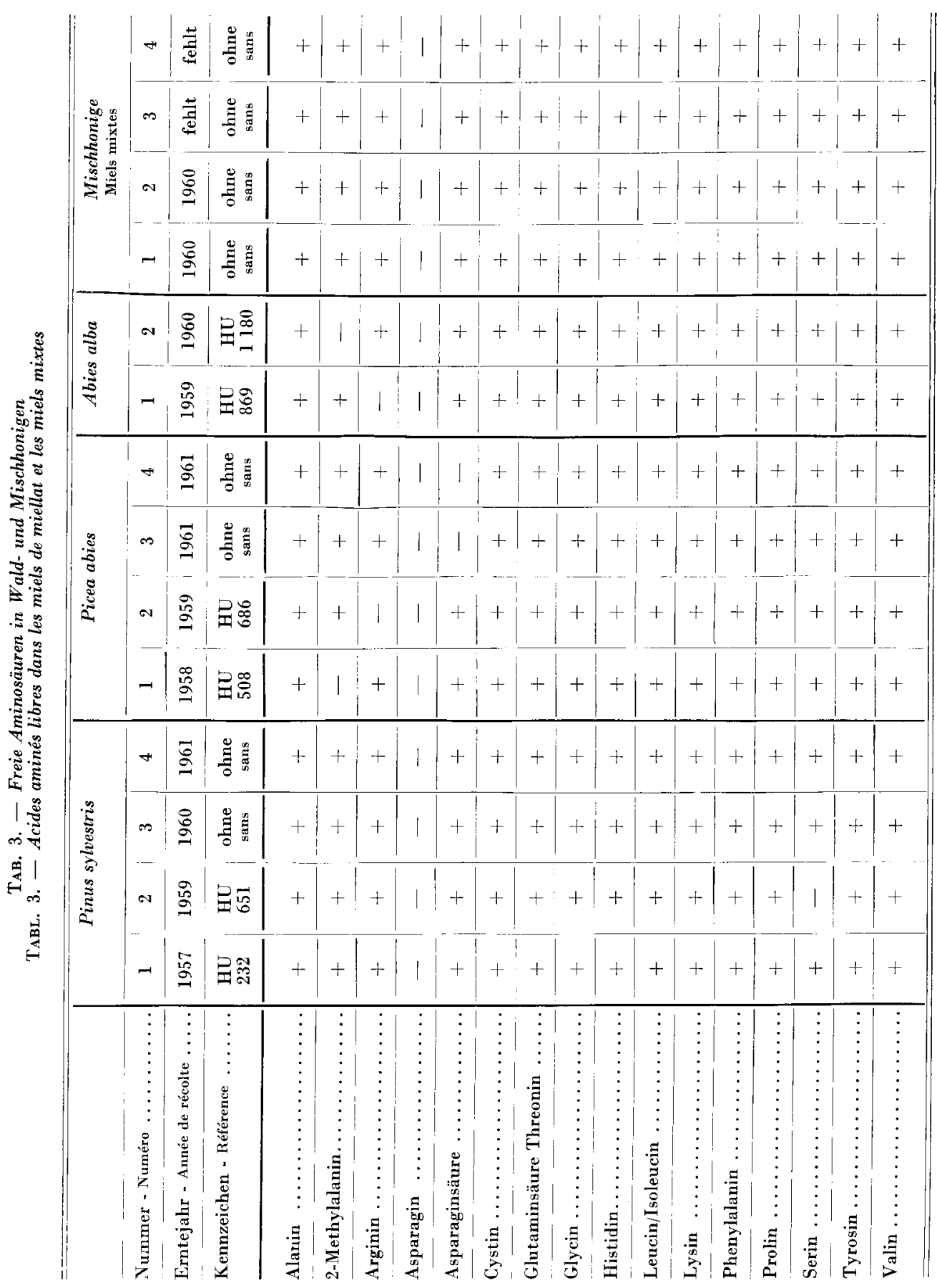


Zu TAB. 3 -.. TABl. 3. - (Explications)

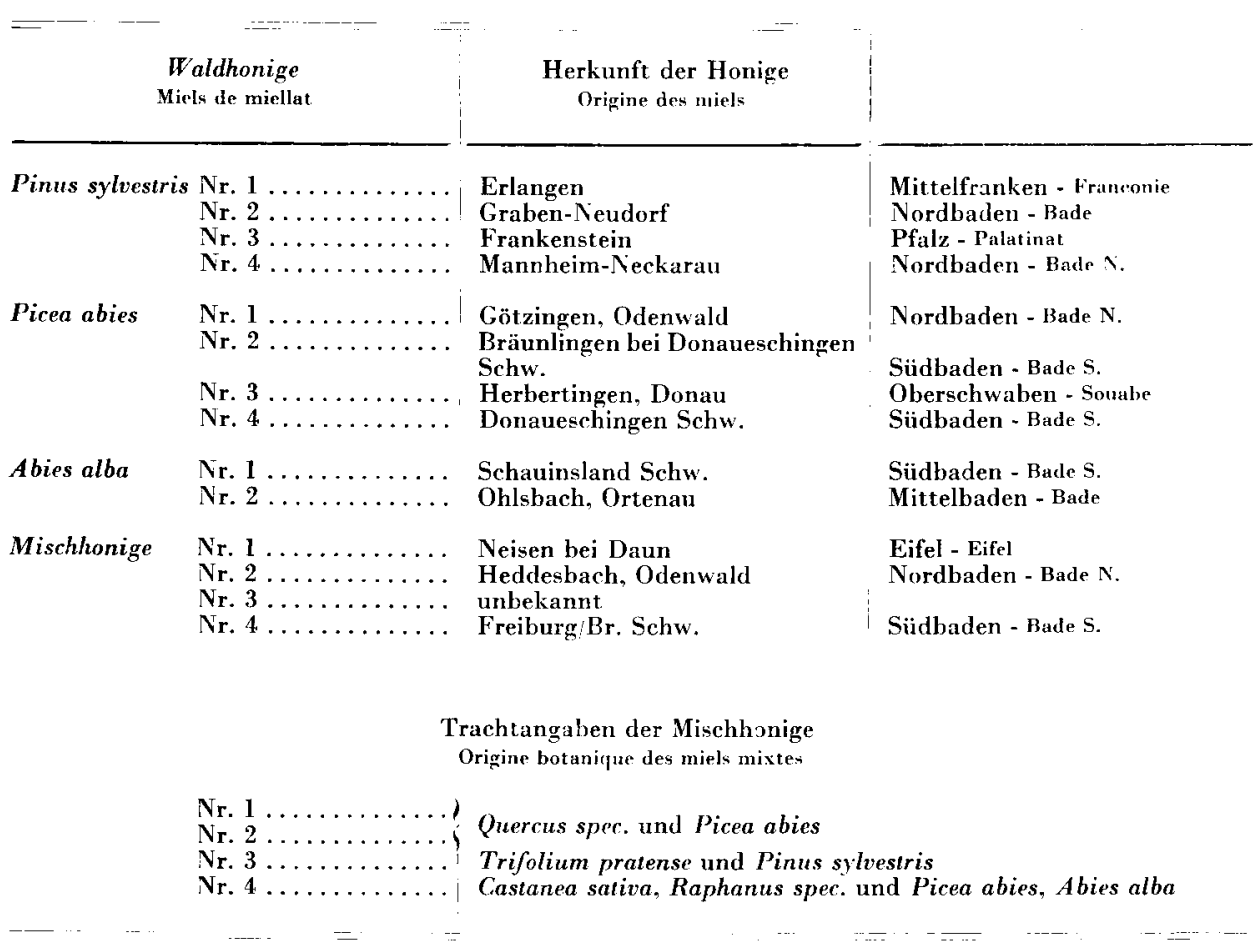

Aus den Waldhonigen (Tab. 3) konnten im Vergleich zu den Blütenhonigen die Aminosäuren in höherer Anzahl und mit verstärkter Farbintensität bestimmt werden. Asparagin allerdings wurde in keinem der Honige gefunden. Bei lo Waldhonigen fehlten in 2 Fällen 2 Methylalanin, Arginin und Asparaginsäure. Serin fehlte in einer Probe.

Die Werte aus dem Bakterizidtest (Tab. 1) sind für 5 von lo Prohen vorhanden; 4 davon zeigen eine höhere antibakterielle Wirkung an als die aufgeführten Blütenhonige, ausgenommen die schon besprochene CastaneaProbe HU 800.

\section{Honige aus Pinus sylvestris-Tracht}

Obwohl nach Herkunft und Erntejahrgängen sehr verschieden (siche Tab. 3), wiesen bis auf das einmalige Fehlen von Serin in Probe HU 651 alle Proben 15 Aminosäuren auf.

\section{Honige aus Picea abies-Tracht}

Die ersten beiden der insgesamt 4 Proben sind aus Götzingen, Nordbaden, aus der Ernte 1958 und aus Bräunlingen bei Donaueschingen, Südbaden, Ernte 
1959. Ihnen fehlte jeweils 1 Aminosäure, nämlich einmal 2- Methyl-alanin, zum anderen Arginin. Die weiteren beiden Honige haben das Erntejahr 1961 und das Fehlen von Asparaginsäure gemeinsam. Geerntet sind sie in Herbertingen / Donau und Donaueschingen, Südbaden. In allen 4. Proben konnten 14 Aminosäuren nachgewiesen werden.

Honige aus Abies alba-Tracht

Einer der beiden Honige wurde 1959 auf dem Schauinsland, Schwarzwald, Südbaden geerntet, es fehlte ihm Arginin. Der andere kam aus Ohlsbach, Ortenau, Mittelbaden aus der Ernte 1960; ihm fehlte 2- Methyl-alanin. In beiden Proben wurden 14 Aminosäuren sichtbar.

\section{4. - Mischhonige}

Mischhonige werden aus den Rohstoffen Nektar und Honigtau von den Bienen bereitet. Asparagin war in den 4 untersuchten Proben nicht nachweisbar, obwohl die Probe Nr. 3 (Tab. 3) aus Trifolium pratense- und Pinus sylvestris- Tracht gemischt ist. Die Proben Nr. 1 und Nr. 2 stammen aus der Ernte 1960, einmal aus Neisen bei Daun, Eifel, zum anderen aus Heddesbach, Odenwald, Nordbaden, aus Quercus spec. -und Picea abies- Tracht. Die Probe Nr. 4 ist aus 4 verschiedenen Trachten gemischt : Castanea sativa, Raphanus spec., Picea abies und Abies alba. Die Probe wurde in Freiburg/Br., Südbaden, geerntet. In allen 4 untersuchten Mischhonigen war das Gesamtspektrum der 15 nachweisbaren Aminosäuren enthalten.

\section{5 - Zuckerfütterungshonig}

Der in einem Flugzelt von Bienen aus Zuckerlösung bereitete “ Honig » enthielt die Aminosäuren Alanin, Arginin, Glycin, Histidin, Lysin, Prolin, Serin (und Tyrosin?). Es fehlten die in allen anderen Honigen vorkommenden Aminosäuren Glutaminsäure/Threonin, Leucin/Isoleucin, Phenylalanin und Valin. Schlüsse auf die Herkunft der Aminosäuren läßt eine Einzelprobe jedoch nicht zu.

\section{6. - Honigtaue}

Zweige mit Lauskolonien wurden in großen Standzylindern im Labor für etwa drei Wochen frisch erhalten. Während dieser Zeit ließ sich auf darunter festgeschraubten Glasplatten der abtropfende Tau sammeln. Von zwei Lachnilen-Arten waren völlig staubfrei Honigtaumengen nur zwischen 1- $2 \mathrm{ml}$ zu 
erhalten. Dies ist ungefähr die Hälfte der Menge, die zur Analysenlösung benötigt wird. Ich setzte darum die Säulen in halber Höhe an, um zu gleichen Ergebnissen zu kommen.

Der Honigtau der Buchenkrebshaumlaus, Lachnus exsiccator Alt., syn. Schizodryobius pallipes Htg. sah glasklar aus; in ihm konnten alle 15 Aminosäuren nachgewiesen werden (ohne Asparagin). Cystin und 2 Methyl-alanin bleiben fraglich, da sie nicht in regelmäßiger Wiederholung erschienen. Prolin war in allen Proben sichtbar, aber wesentlich schwächer als in allen Honigen, obwohl das Gesamtchromatogramm eine starke Färbung aufwies.

Der Honigtau der Zirbelkiefer-Rindenlauss, Cinaria cembrae Chol., sah milchig weiß aus. Er wies außer Asparaginsäure und 2- Methyl-alanin alle übrigen 13 Aminosäuren auf. Das Chromatogramm war ebenfalls ausgeprägt, jedoch Prolin stark sichtbar. Überraschenderweise wurde als 14. Aminosäure Asparagin sichtbar, das bisher nur im Trifolium pratense- Honig nachweisbar war.

Die Zweige sind von der Engadin-Exkursion 1961 mitgebracht worden. Zur Erklärung, daß Zirbelkieferhonigtau Asparagin enthält, könnte folgender Gedankengang beitragen :

Auf den Bergwiesen im Engadin wachsen verschiedene Kleearten, z.B. Trifolium pratense, $T$. montanum (bis $2000 \mathrm{~m}$ hoch), $T$. repens, der nach G. HegI (1925) vom Flachland bis zum Oberengadin (2 $600 \mathrm{~m}$ ) vorkommt. Wenn wir annehmen, daß die Zirbelkiefer durch den Klee auf einem mit Stickstoff angereicherten Boden stand, könnte unter Umständen der Siebröhrensaft Asparagin, das Endprodukt der Stickstoffentgiftung der Pflanze, enthalten (vergleiche Strasburger 1958). Die Quelle der Lausnahrung sind die Siebröhrensäfte, auf diese Weise wäre Asparagin in den Honigtau gelangt.

Dies könnte eine Anregung dafür sein, mehrere Kleearten, Siebröhrensäfte der in der Nähe wachsenden Bäume und den Honigtau der sich auf diesen bildet, nebeneinander zu prüfen.

\section{IV. - DISKUSSION DER ERGEBNISSE}

Zur Zeit der vorliegenden Untersuchung (1961) war mir nur die Arbeit von Baumgarten u. Möckesch (1956), die in Honigen freie Aminosäuren nachwiesen, zugänglich. Im Gegensatz zu den genannten Autoren habe ich jedoch zur qualitativen Analyse andere Kationenaustauscher und papierchromatographische Methoden angewendet. Unabhängig hat Komamine (1960) auf ähnliche Weise handelsüblichen finnischen Honig mit Importhonig verglichen. In letzterem fand er im Einzeltest Asparagin, wie ich im Trifolium pratenseHonig. Leider waren ihm die Nektarquellen der Honige nicht bekannt, wie er 
ausdrücklich betont. Weitere Arbeiten, die Asparagin erwähnen, sind von Curti u. Riganti (1966) und Bergner u. Hahn (1972) veröffentlicht; in ihnen sind die einzelnen Aminosäuren auch quantitativ nachgewiesen. Bei Curti u. Riganti wird in Honigen aus 20 Provinzen Italiens Asparagin mit Serin gemeinsam bestimmt. Serin allein wird bei allen erwähnten Autoren in allen Honigen nachgewiesen, Baumgarten u. Möckesch fanden es jedoch nur in 9 von 15 Proben. Bei Bergner u. Hahn wird im Honigtauhonig des Schwarzwaldes und ebenso in deutschen und ausländischen Blütenhonigen Asparagin mit Glutamin ungetrennt analysiert. Im Gegensatz zu den vorgenannten Autoren habe ich in Waldhonig nie Asparagin gefunden, jedoch sind unsere Ergebnisse wegen verschiedener Analysenmethoden nicht direkt vergleichbar. Glutamin wurde lediglich von Koмамгne und zwar nur im Importhonig gefunden. Durch alle diese Ergebnisse ist meine Aussage, Asparagin nur im Trifolium pratenseHonig analysiert zu haben, mit meinem Hinweis auf ein mögliches Vorkommen in anderen Leguminose-Honigen, weder bestätigt noch widerlegt. Dieser stützt sich einmal auf den Asparagin-Nachweis in den Nektaren von T. pratense und T. repens sowie Vicia spec, zum anderen auf die bekannte Tatsache, daß Leguminosen stickstoffbindende Pflanzen sind, zur Ammoniakentgiftung die Glutaminsäure und die Asparaginsäure brauchen und Endprodukte dieses Vorgangs Glutamin und Asparagin sind (Strasburger 1958). Glutaminsäure wurde von allen genannten Autoren, außer Bergner u. HaHs, in allen Honigproben nachgewiesen; in der vorliegenden Arbeit konnte diese nicht von Threonin abgetrennt werden. Asparaginsäure wurde von Bergner u. HahN, gleich mir, aber im Gegensatz zu den übrigen Autoren, nicht in allen Honigproben gefunden.

2- Methylalanin ( $\alpha$-Amino-iso- buttersäure), meines Wissens in Honig bisher nicht nachgewiesen, fand ich nicht in allen Proben. Arginin fehlte bei mir in einem Teil der Proben, bei Baumgarten u. Möckesch in ca der Hälfte des Untersuchungsmaterials, bei Комамine im Importhonig und bei CurTi $u$. Riganti in allen Analysen; dagegen wird es von Bergner u. Hahn in allen Honigen nachgewiesen.

Zur Unterscheidung von Blüten- und Waldhonig diente innerhalb dieser Arbeit das wechselnde Fehlen der Aminosäuren Cystin, Glycin und Histidin in Blütenhonigen; davon fehlt Cystin allein oder in der Kombination Cystin/ Glycin, am häufigsten jedoch fehlt die Kombination Glycin/Histidin. Cystin findet nur Komamine in allen Proben, sonst wird es als fehlend oder unter der Erfassungsgrenze oder als nicht überall aufgefunden registriert. Glycin wird von Komamine und Curti u. Riganti in allen Proben nachgewiesen, dagegen wird es von den übrigen erwähnten Autoren, gleich mir, nicht ïberall gefunden. Histidin weisen Curti u. Riganti und Bergner u. Hahn in allen Analysen nach, bei Baumgarten u. Möckesch fehlt es teilweise und bei Komamine ganz.

Die Möglichkeit, daß die als fehlend aufgeführten Aminosäuren unter der 
Erfassungsgrenze der angewendeten qualitativen Methode lagen, darf nicht ausgeschlossen werden, zumal Blütenhonige durchweg ein schwächeres Chromatogramm als Waldhonige zeigten. Vielleicht trifft dies auch für Methionin und Tryptophan zu, die ich allerdings in keiner der Proben nachweisen konnte.

Eingegangen im November 1973.

Reçu pour publication en novembre 1973.

\section{DANK}

Diese Untersuchungen wurden auf Veranlassung des Direktors des Forstzoologischen Institutes der Universität Freiburg/Br., Herrn Professor Dr. Dr. G. Wellenstein, 1961 durchgeführt und 1972 ausgearbeitet. Ihm sei mein ganz besonderer Dank dafür ausgesprochen. Zu danken habe ich außerdem Frau Dr. Maurizio, Bern-Liebefeld, Schweiz, für die Überlassung von Honigen und kritische Durchsicht des Manuskriptes, ebenso Herrn Dr. Buchner, Freiburg/Br.- Auch Herrn Professor Dr. Ziegler, München, danke ich für freundliche Unterstützung.

\section{RÉSUMÉ}

Le but des recherches effectuées était de connaître le spectre des acides aminés des miels de fleurs, des miels de miellat et des miels mix tes (miels de fleurs mélangés naturellement à des miels de miellat). A l'origine de la question posée se trouvent les travaux sur l'importance de la fourmi rousse (Formica rufa) pour la production du miellat et l'économie apicole. (Wellenstein, 1958, 1960, 1961).

\section{Méthodes}

Au moment où furent faites ces recherches (1961), seul le travail de BaumGarten et Мöкеsсн (1956) concernant la présence d'acides aminés libres dans le miel nous était accessible. Pour cette raison nous avons transposé des méthodes éprouvées sorties du domaine de la botanique à l'étude de notre matériel expérimental, le miel. Les acides aminés libres sont déterminés qualitativement au moyen de la chromatographie sur papier.

Les solutions à analyser sont préparées à l'aide d'un échangeur d'ions I de Merck (échangeur de cations très acide) et les acides aminés sont élués à l'ammoniaque; la séparation de ces acides se fait par chromatographie sur papier Whatman 1 avec comme solution le butanol/acide acétique glacial/eau dans les proportions $4 / 1 / 5$ et $4 / 1 / 1$.

Avec cette méthode on a pu mettre en évidence 18 acides aminés. Le réactif est une solution de ninhydrine dans l'acétone à $0,5 \%$ avec addition de lutidine $(5 \mathrm{ml}$ pour $100 \mathrm{ml})$.

La sûreté des résultats est basée sur le test 8 à 17 fois positif par acide aminé et par solution à analyser.

Les résultats obtenus figurent dans les tableaux 2 et 3.

La répartition en miels de fleurs, miels de miellat et miels mix tes est faite selon les données fournies avec le matériel.

\section{Résultats}

1) On a mis en évidence dans les miels de fleurs, miels de miellat et miels mixtes examinés les acides aminés suivants : alanine, acide glutamique/thréonine (non séparés), leucine/isoleucine (non séparés), lysine (avec deux exceptions), phénylalanine, proline, sérine (avec 3 exceptions), tyrosine et valine. 
Les acides aminés suivants n'ont été trouvés que dans une partie des miels : 2-méthylalanine, arginine, asparagine, acide aspartique, cystine, glycine, histidine.

2) La méthode analytique permettait de mettre en évidence 18 acides aminés; dans les miels on n'a pu en trouver au maximum que 16; il manquait la méthionine et je tryptophane.

3) On a trouvé une différence entre les miels de fleurs et les miels de miellat; la cystine, la glycine et l'histidine manquent dans les miels de fleurs mais il est rare que ces trois acides aminés manquent à la fois.

4) L'asparagine n'a été trouvée que dans le miel de Trifolium pratense.

5) Pour la première fois on a mis en évidence la 2-méthylalanine (acide $\alpha$-iso-aminobutyrique) dans différents miels.

6) Le nombre maximum d'acides aminés présents dans un miel déterminé est de 15; c'est le cas pour 3 échantillons de miellat de Pinus sylvestris, un échantillon de miel de Castanea sativa et pour tous les miels mixtes.

7) Sur 14 échantillons de miels de fleurs l'analyse a montré la présence, en moyenne, de 12 acides aminés (sans l'asparagine) et 12,5 acides aminés avec l'asparagine; sur 10 échantillons de miel de miellat, 14 acides aminés et sur 4 échantillons mixtes, 15 acides aminés.

\section{LITERATUR}

Baumgarten, F., Möckesch I., 1956. Über die Papierchromatographische Auffindung freier Aminosäuren im Bienenhonig. Z. f. Bienenforsch., 3, 181-184.

Bergner K. G., Hahy Hj., 1972. Zum Vorkommen und zur Herkunft der freien Aminosäuren in Honig. Apidologie, 3, 5-34.

Buchner R., 1966. Vergleichende Untersuchungen über die antibakterielle Wirkung von Blüten-und Honigtauhonigen. Südwestdeutscher Imker, 18, 240-241.

Buchen R., 1967. Über den Untersehied von Bienenhonig aus Blüten-und Honigtautracht. Mitt. bad. Landesver. Naturkd. u. Naturschutz, 9, 589-593.

Cramer F., 1958. Papierchromatographie, Verl. Chemie GMBH., Weinheim/Bergstra ße.

Cunti R., Riganti V., 1966. Ricerche sugli aminoacidi del miele. Rassegna Chimica, N 6, 278-282.

EcklofF W., 1972. Beitrag zur Ökologie und forstlichen Bedeutung bienenwirtschaftlich wichtiger Rindenläuse. Z. f. angew. Entomol. 70, 134-157.

HegI G., 1925. Illustrierte Flora von Mitteleuropa. Lehmanns-Verl., München, 4, 3 ter Teil.

Heilenz S., Höfner W., Neumann K., 1970. Biochemisches Praktikum f. Landwirtschafts-, Forstwirtschafts und Ernährungswissenschaften. Bibliogr. Inst. Mannheim, Wien, Zürich.

Kloft W., Maurizio A., Kaeser W., 1965. Das Waldhonigbuch. Ehrenwirth-Verl., München.

Komamine A., 1960. Amino acids in Honey. Suomen Kemistilehti 33 B, 185-187.

Linskens H. F., 1959. Papierchromatographie in der Botanik. Springer-Verl., Berlin, Göttingen, Heidelberg.

Maurizio A., 1949. Beiträge zur quantitativen Pollenanalyse des Honigs. Beih. Schweiz. Bienenztg., 2, 320-421.

Maurizio A., 1959. Papierchromatographische Untersuchungen an Blütenhonigen und Nektar. Ann. l'A beille, 2, 291-431.

MaUrizio A., 1962. Papierchromatographische Untersuchungen an einigen Honigtau-und Tau-Honigarten. Verh. 11. Int. Kongr. Ent., Wien, 11, 542-543.

Merten B., 1963. Hochspannungselektrophoretische Untersuchungen z. qualitativen u. quantitativen Bestimmung v. Aminosäuren, speziell quantitative Bestimmung von Oxyprolin, Glycin und Lysin zur Ermittlung des Bindegewebsanteils in Fleischerzeugnissen. Diss. Tierhyg. Freiburg/Br. 
Strasburger E., 1958. Lehrbuch der Botanik. Verl. Fischer Stuttgt.

Wellenstein G., 1958. Die Trophobiose der Waldameisen und ihre bienenwirtschaftliche Bedeutung. Verh. d. Dtsch. Ges. f. angew. Entomol,, 14. Vers. 1957, 109-114.

Wellenstein G., 1960. Ergebnisse vierjähriger Untersuchungen über die Steigerung der Waldbienentracht. Z. f. angew. Entomol. 47, 32-41.

Wellenstein G., 1961. Honigtaubildende Forstinsekten und ihre wirtschaftliche Bedeutung. Forstwiss. i. Dienste d. Praxis, Vortrag Forstl. Hochsehulw., Freiburg/Br. 1961, 184-199.

Wellenstein G., 1967. Neunjährige Studien über die Beziehung zwischen Waldameisen, honigtauerzeugenden Insekten und Bienen im Schwarzwald. Wiss., Z. T. Univ. Dresden, 16, 596-598.

Zimmermans, G., o. J. Der Nachweis von Aminosäuren im Siebröhren-und Blutungssaft einiger Bäume. Staatsexamensarbeit (um 1961), Techn. Hochschule Darmstadt. 